\title{
A study of the nuclear structure in the even-even $\mathrm{Yb}$ isotopes
}

\section{A. Zyriliou, ${ }^{a, *}$ T.J. Mertzimekis, ${ }^{a}$ A. Chalil, ${ }^{a, b}$ P. Vasileiou, ${ }^{a}$ E. Mavrommatis, ${ }^{a}$ \\ D. Bonatsos, ${ }^{c}$ A. Martinou, ${ }^{c}$ S. Peroulis ${ }^{c}$ and N. Minkov ${ }^{d}$}

${ }^{a}$ Department of Physics, University of Athens, Zografou Campus, GR-15784, Athens, Greece

${ }^{b}$ IRFU, CEA, Universit'e Paris-Saclay, FR-91190, Paris, France

${ }^{c}$ Institute of Nuclear and Particle Physics, National Center for Scientific Research "Demokritos", GR-15310, Aghia Paraskevi, Greece

${ }^{d}$ Institute of Nuclear Research and Nuclear Energy, Bulgarian Academy of Sciences, 72 Tzarigrad Road, 1784, Sofia, Bulgaria

E-mail: kzyriliou@phys.uoa.gr

The nuclear properties of the Ytterbium isotopes and their evolution as the neutron number increases has been a central objective in this work. The medium-to-heavy mass Yb isotopes are known to be well-deformed rotational nuclei which can be populated to very high spins. Spectroscopic information becomes scarcer for neutron-rich nuclei, impeding the understanding of nuclear structure in this mass region, where interesting phenomena, such as shape coexistence [1], have been predicted to exist. The collective behavior of the even-even ${ }^{164-180} \mathrm{Yb}$ isotopes was investigated using several well-established theoretical models in synergy with available experimental data. In this work, reduced transition probabilities $B(E 2)$ and transition quadrupole moments $Q$ for even-even ${ }^{164-178} \mathrm{Yb}$ isotopes have been calculated using the Interacting Boson Model(IBM-1).

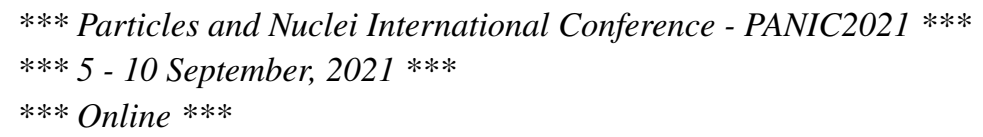

${ }^{*}$ Speaker 


\section{Interacting Boson Model (IBM-1)}

The Interacting Boson Model (IBM) of Arima and Iachello [2] has been employed to make a schematic study of the $\mathrm{Yb}$ isotopes. The main idea of the IBM is to describe the low-lying collective states of several medium- and heavy-mass nuclei. In this work we apply the model to the even-even $\mathrm{Yb}$ isotopes $(\mathrm{Z}=70,94 \leq N \leq 108)$. For each $\mathrm{Yb}$ isotope the values of the five parameters in the IBM Hamiltonian (Eq. 1), have been determined, so as to obtain the best fit to the available experimental data. The number of bosons is counted from the nearest closed shell. The total number of bosons, $N_{B}$, which in the IBM-1 could have been considered as a parameter, is now fixed by the microscopic interpretation of the bosons to be $N_{B}=N_{\pi}+N_{v}$, where $N_{\pi}$ and $N_{v}$ are the proton and neutron boson numbers, respectively. For the IBM calculations we used the extended consistent $Q$ formalism (ECQF) [3], which has been introduced in [4, 5], with the Hamiltonian [6, 7],

$$
H(\zeta)=c\left[(1-\zeta) \hat{n}_{d}-\frac{\zeta}{4 N_{B}} \hat{Q}^{\chi} \cdot \hat{Q}^{\chi}\right],
$$

where $\hat{n}_{d}=d^{\dagger} \cdot \tilde{d}$ is the quadrupole boson creation operator, $N_{B}$ is the number of bosons, $c, \zeta$ are free parameters and $\hat{Q}^{\chi}=\left(d^{\dagger} s+s^{\dagger} \tilde{d}\right)+\chi\left(d^{\dagger} \tilde{d}\right)^{(2)}$ is the quadrupole operator. The combinations of $\varepsilon=c(1-\zeta)$ and $\kappa=-c\left(\zeta / 4 N_{B}\right)$ are also used.

For each $N_{B}\left(N_{B}=12-17\right)$ nearly 20,000 calculations have been carried out with the code IBAR [8]. Results were obtained by fitting five observables to experimental data available for each isotope. Since we consider only ratios for the $E 2$ transition rates, the effective boson charge $e_{B}$ cancels out.

It is well known $[9,10]$ that specific observables can describe the low-spin structure of the collective even-even nuclei. The most useful are in a notation $R_{4 / 2}=E\left(4_{1}^{+}\right) / E\left(2_{1}^{+}\right), E\left(0_{2}^{+}\right) / E\left(2_{1}^{+}\right)$, $E\left(2_{\gamma}^{+}\right) / E\left(2_{1}^{+}\right)$, where the $2_{\gamma}^{+}$state is the bandhead of the quasi- $\gamma$ band in rotational nuclei. Also for the electromagnetic transition probabilities, the $B(E 2)$ ratio $B_{2 \gamma}=B\left(E 2 ; 2_{\gamma}^{+} \rightarrow 0_{1}^{+}\right) / B\left(E 2 ; 2_{1}^{+} \rightarrow\right.$ $\left.0_{1}^{+}\right)$, as well as the branching ratio, $R_{2 \gamma}=B\left(E 2 ; 2_{\gamma}^{+} \rightarrow 0_{1}^{+}\right) / B\left(E 2 ; 2_{\gamma}^{+} \rightarrow 2_{1}^{+}\right)$, are useful [9]. These observables were used as they are relatively straightforward to measure and involve the bandheads that are relatively simple to identify. The method leaves $\zeta$ and $\chi$ as free parameters in the range (0.00 to 1.00$)$ for $\zeta$ and $(-\sqrt{7} / 2$ to 0.00$)$ for $\chi$ with step 0.01 , where $\chi=-\sqrt{7} / 2$ is the limit of $\mathrm{SU}(3)$ symmetry.

The IBM calculations can provide results for a large number of observables. These values can be used to gain information for the shape of the nucleus. Generally it is sufficient to use only the deformation parameter $\beta_{2}>0$ and $0^{\circ}<\gamma<60^{\circ}$ to describe the nuclear shape, because for every set of parameters outside this range, it is possible to find parameters inside this range which describe the same shape of the nucleus, with only the orientation in the coordinate system being different.

Figure 1 shows the numerical results for the IBM predictions for $\gamma$, compared to experimental values [11], extracted from ratios of the $\gamma$ bandhead to the first $2^{+}$state, according to $[1,12,13]$,

$$
\begin{gathered}
R=\frac{E\left(2_{\gamma}^{+}\right)}{E\left(2_{1}^{+}\right)} \\
\sin (3 \gamma)=\frac{3}{2 \sqrt{2}} \sqrt{1-\left(\frac{R-1}{R+1}\right)^{2}} .
\end{gathered}
$$




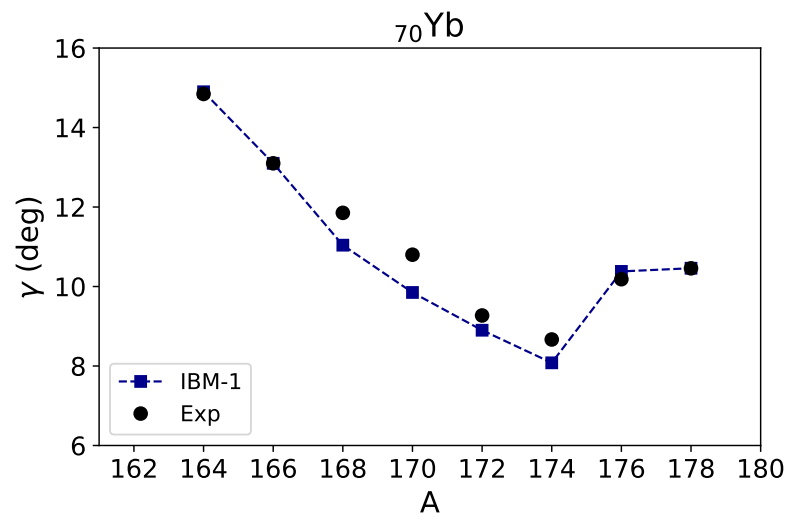

Figure 1: IBM predictions for $\gamma$, obtained from Eq. (3), compared to experimental values extracted from ratios of the $\gamma$ bandhead to the first $2^{+}$state. Experimental uncertainties are smaller than the data symbols.

\section{Results}

We present the calculated values of $B\left(E 2 ; 0_{1}^{+} \rightarrow 2_{1}^{+}\right)$and quadrupole moments $Q$ and compare them with the experimental ones [11]. For IBM the results have been calculated directly from the model.
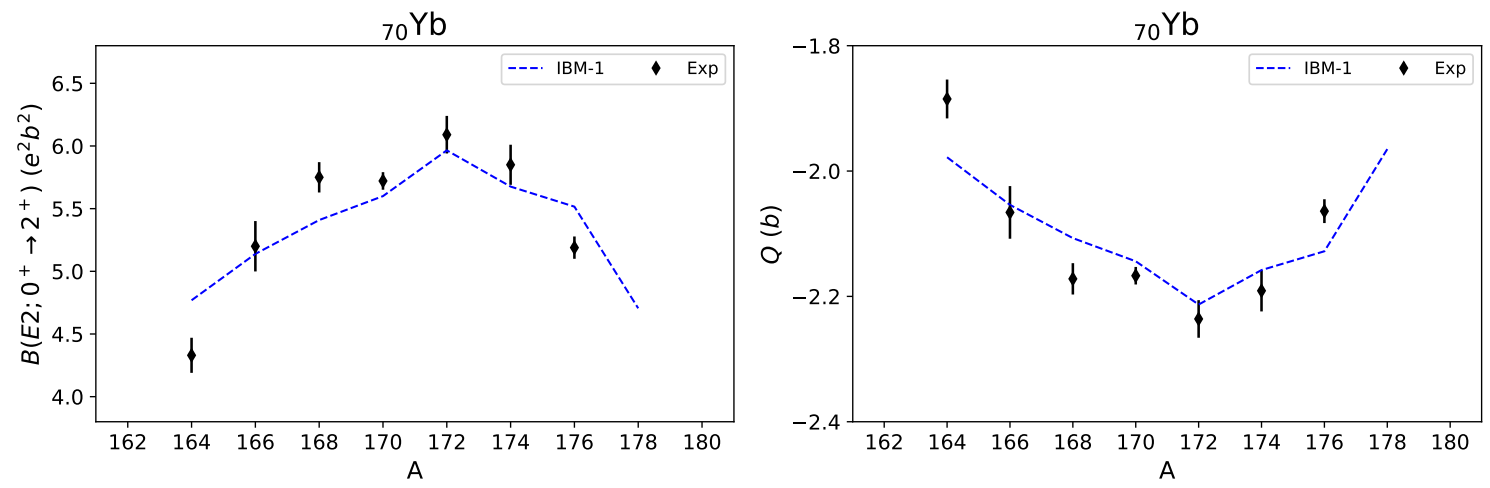

Figure 2: Reduced transition probabilities $B\left(E 2 ; 0^{+} \rightarrow 2^{+}\right)$values and electric quadrupole moments $Q$ values for IBM-1 are compared with experimental ones [11]. Maximum deformation is observed in ${ }^{172} \mathrm{Yb}$, two neutrons away from the mid-shell closure. The blue dashed lines are used to guide the eye.

\section{Conclusion \& Future Directions}

In the framework of the nuclear collective model, the nuclear observables examined in the present work for a number of permanently deformed prolate $\mathrm{Yb}$ isotopes are calculated and shown to be in good agreement with available experimental data. This work can serve as a reference point for future experimental and theoretical work in this mass region, which will provide useful information towards understanding the nuclear structure as one moves closer to the neutron dripline. 


\section{Acknowledgments}

This research work was supported by the Hellenic Foundation for Research and Innovation (HFRI) under the HFRI PhD Fellowship grant (Fellowship Number: 101742/2019) for AZ.

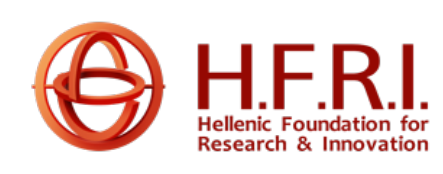

\section{References}

[1] D. Bonatsos, I.E. Assimakis, N. Minkov, A. Martinou, R.B. Cakirli, R.F. Casten et al., Proxy-SU(3) symmetry in heavy deformed nuclei, Phys. Rev. C 95 (2017) 064325.

[2] A. Arima and F. Iachello, Interacting boson model of collective states I. The vibrational limit, Ann. Phys. 99 (1976) 253.

[3] P.O. Lipas, P. Toivonen and D.D. Warner, IBA consistent-Q formalism extended to the vibrational region, Phys. Lett. B 155 (1985) 295.

[4] D.D. Warner and R.F. Casten, Revised Formulation of the Phenomenological Interacting Boson Approximation, Phys. Rev. Lett. 48 (1982) 1385.

[5] D.D. Warner and R.F. Casten, Predictions of the interacting boson approximation in a consistent $Q$ framework, Phys. Rev. C 28 (1983) 1798.

[6] N.V. Zamfir, P. von Brentano, R.F. Casten and J. Jolie, Test of two-level crossing at the $N=90$ spherical-deformed critical point, Phys. Rev. C 66 (2002) 021304.

[7] V. Werner, P. von Brentano, R.F. Casten, C. Scholl, E.A. McCutchan, R. Krücken et al., Alternative interpretation of E0 strengths in transitional regions, Eur. Phys. J A - Hadrons and Nuclei 25 (2005) 455.

[8] R.J. Casperson, IBAR: Interacting boson model calculations for large system sizes, Comput. Phys. Commun. 183 (2012) 1029.

[9] W.-T. Chou, N.V. Zamfir and R.F. Casten, Unified description of collective nuclei with the interacting boson model, Phys. Rev. C 56 (1997) 829.

[10] E.A. McCutchan, N.V. Zamfir and R.F. Casten, Mapping the interacting boson approximation symmetry triangle: New trajectories of structural evolution of rare-earth nuclei, Phys. Rev. C 69 (2004) 064306.

[11] B. Pritychenko, M. Birch, B. Singh and M. Horoi, Tables of E2 Transition Probabilities from the first $2^{+}$States in Even-Even Nuclei, Atom. Data Nucl. Data Tabl. 107 (2016) 1.

[12] A.S. Davydov and G.F. Filippov, Rotational states in even atomic nuclei, Nuclear Physics 8 (1958) 237.

[13] L. Esser, U. Neuneyer, R.F. Casten and P. von Brentano, Correlations of the deformation variables $\beta$ and $\gamma$ in even-even Hf, W, Os, Pt, and Hg nuclei, Phys. Rev. C 55 (1997) 206. 